\title{
From millimeter to microns - IAP RAS powerful sources for various applications
}

\author{
M. Yu. Glyavin, G. G. Denisov, E.A. Khazanov \\ Institute of Applied Physics RAS, Nizhny Novgorod, Russia, glyavin@appl.sci-nnov.ru
}

The last decade has contributed to the rapid progress in the development of $\mathrm{THz}$ sources, in particular gyrotrons. Although in comparison with the classical microwave tubes the gyrotrons are characterized by greater volume and weight due to the presence of bulky parts (such as superconducting magnets and massive collectors where the energy of the spent electron beam is dissipated) they are much more compact and can easily be embedded in a sophisticated laboratory equipment (e.g. spectrometers, technological systems, etc.) than other devices with a comparable value of $\mathrm{Pf}^{2}$ such as free-electron lasers (FELs) and radiation sources based on electron accelerators. All these advantageous features have opened the road to many novel and prospective applications of gyrotrons as radiation sources in a great number of high power $\mathrm{THz}$ technologies, advanced spectroscopic techniques, plasma science and materials processing, fusion research as well as in many other scientific and technological fields.

The gyrotron developed at IAP RAS jointly with GYCOM Ltd (Nizhny Novgorod) provides CW radiation with a power from $1 \mathrm{~W}$ up to $300 \mathrm{~kW}$ in the frequency range $0.26-0.52 \mathrm{THz}[1,2]$ and up to $1 \mathrm{MW}$ at frequencies $0.14-0.17 \mathrm{THz}$ [3]. It is important, that power up to $10 \mathrm{~W}$ needed for spectroscopy applications can be obtained at low voltage about $1.5 \mathrm{kV}$ or low beam current - tens $\mathrm{mA}$.

The experiment on frequency stabilization was carried out using a continuous-wave $(\mathrm{CW})$ gyrotron operating at a frequency of $263 \mathrm{GHz}$ and an output power of up to $1 \mathrm{~kW}$ with an electron beam formed by a triode-type magnetron injection gun. The phase lock loop control of anode voltage has been used and the width of the frequency spectrum was decreased to 1 $\mathrm{Hz}$ [4], which corresponds $\Delta f / f=3 * 10^{-12}$ with measurement time of a few seconds. The long-term stability was defined by reference clock $\left(\delta f / f \sim 10^{-9}\right.$ for quartz clock and up to $\delta f / f \sim 10^{-12}$ for rubidium clock).

It is interesting, that the same scheme can be used for quick power modulation and data transmitting. Experiment was made based on $1 \mathrm{~kW} / 0.26 \mathrm{THz}$ tube and linear dependence between control (anode) voltage and output frequency was observed. As a result, high quality transmission of sound and pseudo random bit sequence (with a speed up to $1.5 \mathrm{Mbit} / \mathrm{s}$ ) has been obtained $[3,5]$.

The prototype of CW $250 \mathrm{GHz} / 200 \mathrm{~kW}$ gyrotron has been successfully developed. The power up to 300 $\mathrm{kW}$ in 40 microsecond pulses with efficiency more than $30 \%$ was demonstrated [6] with a full accordance with theoretical estimation. The gas discharge experiments, initiated by mentioned microwaves, demon- strate unique plasma parameters. The installation diamond window, needed for CW tests, is in progress.

The gyrotrons with pulsed magnetic fields operating at the fundamental harmonic produce up to 100 $\mathrm{kW}$ power level at $0.7 \mathrm{THz}$ [7] and $1 \mathrm{~kW}$ at $1 \mathrm{THz}$ frequency [8]. The future rising of operating frequency (especially excitation of high harmonics due to limit of reasonable magnetic field) needs improved methods of mode selection, which can be divided to electron-optical and electro-dynamical ones. The most successful realization of electron-optical methods is a gyrotron with axis-encircling electron beam (see, for example [9]). The paper [9] presents the pulsed third harmonic $1 \mathrm{THz}$ tube with a power level several hundred Watts. The CW version of LOG based on the cryomagnet is under tests at the moment.

The second harmonic CW gyrotron with improved mode selection based on double electron beam has been tested [10] jointly with FIR UF (Fukui, Japan). The wide step tuning of frequency by excitation of various modes was demonstrated in the range 0.4$0.75 \mathrm{THz}$. The stable operation at the frequency 0.76 $\mathrm{THz}$ on the second harmonic with power level about few Watts has been obtained, which useful for modern NMR/DNP spectroscopy applications.

The feasibility of a high-power sub-THz gyrotron with smooth wideband frequency tuning suitable for direct measurement of the positronium hyperfine structure is demonstrated numerically using both averaged equations and PIC-code simulations. Analytical estimates show that the frequency tuning through the excitation of axial modes can be achieved in a gyrotron with a short cavity driven by an electron beam with a high current. Simulations [11] and preliminary experiments demonstrates possibility of wide (about 10\%) fine frequency tuning. An output power of $0.5-1 \mathrm{~kW}$ can be obtained at a frequency of about $0.2 \mathrm{THz}$ within a $10-\mathrm{GHz}$ band, which are the parameters needed for testing of quantum electrodynamics predictions through the spectroscopy of positronium.

The pulsed gyrotrons have been used successfully for initiation of localized gas discharges. Such a plasma is promising for development of both a point source of multi-charged ions and a source of highenergy ultraviolet (extreme ultraviolet EUV or XUV) $[12,13]$. The gas discharge has been successfully obtained also with $0.26 \mathrm{THz} / \mathrm{CW}$ IAP RAS gyrotron mentioned above.

The gyrotron looks as a most promising source for the high-resolution molecular spectroscopy in a gas mixture. A significant improvement of spectrum quality due to power growth in contrast with traditional BWO has allowed observing the theoretically 
predicted earlier transitions in $\mathrm{SO}_{2}$ molecule [14]. Sensitivity of a radio-acoustic detector was increased about three orders due to high power. It is important to mention, that during last experimental company we use not only power on fundamental harmonic, but also gyrotron radiation produced due to nonlinear effects at high harmonic simultaneously with the fundamental. Though the generated power at the second and third harmonics is two orders and three orders, respectively, lower than at the first one, such a power level $(1-10 \mathrm{~W})$ is significantly higher than in other sources. In this experiment, the $\mathrm{SO}_{2}$ spectrum line was measured at the frequency range $0.26-0.8 \mathrm{THz}$.

One more interesting feature of powerful sub$\mathrm{THz}$ radiation is high speed production of pure nanopowders by material evaporation and condensation. The use of a subterahertz gyrotron setup with output frequency of $263 \mathrm{GHz}$ and a nominal power of $1 \mathrm{~kW}$ as a radiation source to obtain nanoscale particles of metal oxides by the evaporation-condensation technique is demonstrated. An increase in the substance evaporation rate by more than three times compared with similar experiments using a technological gyrotron setup with a frequency of 24 $\mathrm{GHz}$ and a nominal power of $7 \mathrm{~kW}$ (the power density on the sample surface was about $13 \mathrm{~kW} / \mathrm{cm}^{2}$ ) as a radiation source was demonstrated. An increase in the radiation frequency leads to the improvement of the heating efficiency due to an increase of the absorption coefficient and provides a greater power density due to a better focusing of the radiation [15].

Despite the requirement for strong operating magnetic fields, mode competition, high ohmic losses, etc., the $\mathrm{THz}$ frequency range has been already achieved both by the pulse and $\mathrm{CW}$ gyrodevices. A number of applications rapidly increase with the development of radiation sources.

The basic distinguishing feature of the IAP RAS approach to creating powerful femtosecond laser complexes is the use of optical parametric chirped pulse amplification (OPCPA) instead of conventional laser amplification. The femtosecond laser complex PEARL created at IAP RAS is based on these principles. This complex generate pulses with power up to $0.56 \mathrm{PW}$. The experience gained in creating PEARL and its principles are currently used for constructing at IAP RAS of the laser complex PEARL-10 with a power over $5 \mathrm{PW}$. The concept of an exawatt laser system - the XCELS project [16] has been developed. Appearance of such sources opens up absolutely new horizons for basic research and unique applications. Generation of high-energy, highly collimated bunches of charged particles and creation of secondary sources of powerful X-ray and gamma radiation during the interaction of superintense laser radiation with matter are the most topical problems of modern science. Present-day high-power laser complexes can lay the basis of a new compact technology of particle acceleration and generation of coherent X-ray and gamma radiation. Such technologies have great promise for use in diverse fields of science and engineering. In medicine, high-energy bunches of protons and ions are used for therapy of a variety of cancers. Electron bunches can be used for injection into free-electron lasers.

Acknowledgements. The gyrotron development program supported by the RSF grant 14-12-00887.

\section{References}

1. M.Glyavin et al. High-power terahertz sources for spectroscopy and material diagnostics // PhysicsUspekhi, 59, 6, 595-604 (2016)

2. M.Yu. Glyavin et al. Experimental tests of 263 $\mathrm{GHz}$ gyrotron for spectroscopy applications and diagnostic of various media // Rev. Sci. Instr., 86(5), 054705 (2015)

3. G.Denisov. New development of Megawatt power gyrotrons in Russia // 20th Joint Workshop on Electron Cyclotron Emission and ECRH, Greifswald, Germany. May 14-17, 2018.

4. A.Fokin et al. High power sub-terahertz microwave source with record frequency stability up to $1 \mathrm{~Hz} / /$ Scientific Reports 8, 4317 (2018)

5. A.Fokin, A.Tsvetkov, private communication

6. G.G.Denisov et al. First experimental tests of powerful $250 \mathrm{GHz}$ gyrotron for future fusion research and CTS diagnostics // Rev.Sci.Instr. 89(8), 084702 (2018). DOI:10.1063/1.5040242

7. V.L. Bratman et al. Gyrotron Development for High Power THz Technologies at IAP RAS // $J$ Infrared Millimeter and Terahertz Waves, 33(7), 715-723 (2012). DOI: 10.1007/s10762-012-9898-6

8. M.Yu.Glyavin et al. Experimental Study of the Pulsed Terahertz Gyrotron with Record-Breaking Power and Efficiency Parameters // Radiophysics and Quantum Electronics, 56, 8-9, 497-507 (2014)

9. V.L.Bratman et al. Large-Orbit Gyrotron Operation in the Terahertz Frequency Range // Phys. Rev. Lett. 102, 245101 (2009).

10. T.Idehara et al. A Novel THz-Band DoubleBeam Gyrotron for High-Field DNP-NMR Spectroscopy // Rev. Sci. Instr., 88, 094708 (2017).

11. A.E.Fedotov et al. Frequency Tunable sub$\mathrm{THz}$ Gyrotron for Direct Measurements of Positronium Hyperfine Structure // IEEE Trans. On Electr. Devices, 46, 7, 2465-2469 (2018).

12. M.Yu.Glyavin et al. A point-like source of extreme ultraviolet radiation based on a discharge in a non-uniform gas flow, sustained by powerful gyrotron radiation of terahertz frequency band // Applied Physics Letters 105, 174101 (2014).

13. A.Sidorov et al. Gas discharge powered by the focused beam of the high-intensive electromagnetic waves of the terahertz frequency band // Journal of Physics D: Applied Physics (2018).

14. M.A.Koshelev et al. Molecular gas spectroscopy using radioacoustic detection and high-power coherent subterahertz radiation sources // Journal of Molecular Spectroscopy, 331, 9-16 (2017).

15. A.V.Vodopyanov et al. Application of the $263 \mathrm{GHz} / 1 \mathrm{~kW}$ gyrotron setup to produce a metal oxide nanopowder by the evaporation-condensation technique // Vacuum, 145, 340-346, (2017).

16. www.iapras.ru/english/science/las phys/gen lasf4.html (Online available 01/10/2018) 